\title{
A Noção Transcultural de Maturidade Vocacional na Teoria de Donald Super
}

\author{
Marcos Alencar Abaide Balbinotti ${ }^{1}$
}

Universidade do Vale do Rio dos Sinos

\begin{abstract}
Resumo
Donald Super engajou-se a sistematizar uma teoria geral da escolha e do desenvolvimento vocacional estruturada no modelo tradicional, no de fatores sócioeconômicos e ambientais, no de abordagens desenvolvimentistas, e no fenomenológico. O presente trabalho objetiva apresentar o conceito de maturidade vocacional e sua generalização no plano transcultural. Com este propósito, explorou-se, mais especificamente, o terceiro modelo teórico, aquele do cumprimento, por parte do indivíduo, de etapas ordenadas e previsíveis durante toda sua vida, visto que a noção de maturidade vocacional se revela através da passagem de um processo de desenvolvimento. Através de dados colhidos por diversos inventários de maturidade, apresentou-se os dados relativos a sua generalização. Concluiu-se que o modelo teórico explicativo da maturidade vocacional parece ser aplicável a diversas populações que possuem elementos culturais comparáveis aos americanos. Algumas exceções ou cuidados na interpretação de dados se aplicam. Novos estudos devem ser realizados a fim de melhorar as qualidades psicométricas das medidas de maturidade vocacional em alguns outros países e, também, as adaptações de tais instrumentos levando-se em conta a diferenças sócio-econômicas e culturais.

Palavras-chave: Desenvolvimento vocacional; maturidade vocacional; Teoria de Donald Super; transcultural.
\end{abstract}

The Transcultural Aspect of Vocational Maturity in Donald Super Theory

Abstract

Donald Super conceptualized a general theory of developmental vocational and career choice based on four models: 1) the traditional, 2) the developmental, 3) the economic, social and environmental, and 4) the phenomenological. Considering that vocational maturity can be revealed by the constant process of interaction between individuals and their environment, this present work aims to explore the concept of vocational maturity within the transcultural field (the third model). Data were collected through several maturity inventories and results show that Super's theoretical model of vocational maturity can be generalized to several cultures, in particular to the ones that can be comparable to the American society, although some careful data examination is always needed. Finally, we suggest the need of new studies aiming to improve the psychometric properties of vocational measures in some countries, trying to pinpoint cultural and social differences when they appear.

Keywords: Vocational development; vocational maturity; Donald Super's Theory; transcultural.

Para se poder entender, adequadamente, a noção de maturidade vocacional de Donald Super, é conveniente situá-la dentro do contexto geral de sua teoria da escolha e do desenvolvimento vocacional, permitindo uma melhor precisão no sentido que Super dá a este conceito. Em seguida, apoiando-se em algumas pesquisas empíricas importantes, poder-se-á apreciar em que sentido esta noção é generalizável a diferentes culturas.

\section{A Noção de Maturidade Vocacional no Contexto} da Teoria de Donald E. Super

Partindo do histórico estudo de Buehler (1933) sobre o ciclo vital como um aspecto psicológico, a noção de maturidade vocacional, remete, necessariamente, a um processo de desenvolvimento (Balbinotti \& Tétreau, 2002), como todos os outros aspectos da personalidade humana (social, emocional, sexual, intelectual, etc.) com

${ }^{2}$ Endereço para correspondência: Av. Unisinos, 950, Núcleo de Orientação Vocacional, 93022 000, São Leopoldo, RS. Fone: (51) 5908127. E-mail balbinotti@bios.unisinos.br os quais se associa o termo maturidade. Em 1942, em um volume intitulado The Dinamics of Vocational Adjustment, Super apresentou uma síntese do que se conhecia naquela época sobre a escolha de uma carreira. Naquela ocasião, ele propôs uma concepção de escolha profissional que orientou, conseqüentemente, todos os passos teóricos e empíricos de sua carreira (Super, 1951, 1953, 1955, 1957, 1972, 1980 , 1981, 1985a, 1985b, 1990; Super \& Kidd, 1979; D. Super, Savickas \& C. Super, 1996; D. Super, Sverko \& C. Super, 1995). Com efeito, considerando a escolha vocacional como resultado de uma série de decisões pré-vocacionais e vocacionais que podem (ou tendem) durar toda a vida, sua concepção contrastava radicalmente com aquela que havia prevalecido até aquele momento e que se associava com a teoria dos traços e fatores de Parsons (1909).

Naquela época (Parsons, 1909), concebia-se a escolha profissional como sendo um comportamento de acontecimento único e estático na vida de um indivíduo, emparelhando as características pessoais e de personalidade de um sujeito às de uma profissão. As concepções teóricas de caráter desenvolvimentista de Super (1942) foram 
resgatadas por Ginzberg, Ginsburg, Axelrad e Herma (1951) - que finalmente limitaram sua descrição do desenvolvimento vocacional do nascimento a adolescência (ou até o início da idade adulta) -, por Tiedeman (Tiedeman \& O’Hara, 1963 Tiedeman \& Miller-Tiedeman, 1984), e, também, mais recentemente, por Vondracek e colaboradores (Vondracek, Lerner \& Schulenberg, 1986). Tais concepções tornaram-se as principais idéias ou tendências, pareadas àquela de Holland (1959, 1997), compondo as orientações teóricocontemporâneas básicas e de prática em psicologia da escolha e do desenvolvimento profissional. É claro que diversas outras teorias, não menos importantes (embora ainda pouco divulgadas), têm sido elaboradas, estruturadas e aplicadas por diversos autores (Blau, 1993; Breton, McDonald \& Richer, 1972; Brooks, 1984; Farmer, 1984 1997; Havighurst, 1952, 1964, 1972; Knefelkamp \& Slepitza, 1976; Levinson, Darrow, Klein, Levinson \& McKee, 1976, 1978; Miller \& Form, 1951; Riverin-Simard, 1977, 1981, 1984, 1990, 1993, 1996a, 1996b, 1996c, 1998 Roe, 1956, 1957; Roe \& Lunneborg, 1990), mas a de Super ocupa um lugar de destaque, ao menos, quanto ao número constante e atual de publicações em revistas especializadas.

Super $(1969,1990)$ propôs uma concepção de escolha profissional com base em conceitos (maturidade, interesses, valores, etc.) que indicam um processo de desenvolvimento (Buehler, 1933) e que, portanto, contrastava com a simples teoria dos traços e fatores (Parsons, 1909), e ainda incluiu outros modelos que, segundo ele próprio e seus colaboradores (Super, Sverko \& Super, 1995; Super \& cols. 1996), explicariam melhor a complexidade do comportamento vocacional de um indivíduo. Seriam, então, ao todo, quatro modelos que, juntos, teriam a árdua tarefa de desvendar, ao menos em parte, este comportamento São eles: o modelo de perspectiva diferencial, o modelo socioeconômico e ambiental, o modelo desenvolvimentista e, finalmente, o modelo fenomenológico. Uma descrição mais detalhada e objetiva destes modelos será apresentada abaixo.

1) $O$ primeiro modelo, relacionado com as concepções teóricas de Parsons (1909), apóia-se numa psicologia diferencial dos indivíduos e das ocupações ${ }^{2}$. É o modelo tradicional que Holland (1959, 1973, 1985, 1992, 1996, 1997) representa em sua forma contemporânea e segundo o qual busca-se assegurar o bomem certo no lugar certo a partir de uma análise das características do indivíduo e da profissão considerada.

${ }^{2}$ Ocupação é um anglicismo freqüentemente utilizado para falar da profissão ou do trabalho.
2) O segundo modelo diz respeito à influência dos fatores socioeconômicos, tecnológicos e ambientais (tais como a família, a escola, a comunidade, o grupo de pares, a sociedade, a economia, o mercado de trabalho, as políticas sociais e as experiências profissionais) sobre o desenvolvimento de carreira (Bergeron, 1998; Bingham, 1997; Brown, Fukunaga, Umemoto \& Wicker, 1996; Fouad, 1994; Herr \& Lear, 1984; Hotchkiss \& Borow, 1996; Lapointe, 1998; Miller \& Weeks, 1985; Schultz \& Henderson, 1985; Watkins \& Subich, 1995). Por exemplo, segundo Super (1957), a família contribui, no desenvolvimento das necessidades e dos valores, fornecendo à criança, ou ao adolescente, a possibilidade de adquirir informações e desenvolver habilidades que poderão ter uma importante influência no momento da tomada de decisão profissional por parte dos jovens. O autor apresenta também, uma discussão dos fatores econômicos ligados ao desenvolvimento profissional: a lei da oferta e da procura, a tradição, os sindicatos e as organizações profissionais, o custo dos estudos, os ciclos econômicos, a tecnologia e, finalmente, a automatização. Estas, entre outras, são as variáveis que intervêm influenciando a carreira dos indivíduos, tanto no início do exercício de uma ocupação, quanto nas mudanças e adaptações que poderão se impor no decurso dos anos. Recentemente, Osipow e Fitzgerald (1996) concluem que a influência da classe social (média ou elevada) é quase sempre positiva sobre o desenvolvimento vocacional. Segundo estes autores, a classe social não influencia somente a disponibilidade dos recursos em relação à escolha e à adaptação profissionais, mas ela tem, ainda, um efeito sobre as atitudes, os hábitos e aspirações que constituem o contexto sócio-psicológico do desenvolvimento de carreira. Este contexto compreende, por exemplo, os encorajamentos que uma pessoa recebe e as experiências de trabalho que ela conhece. As conclusões de Young e Chen (1999), concernentes à influência da classe social sobre o desenvolvimento de carreira, apóiam a afirmação desses autores.

3) O terceiro modelo, o da psicologia das carreiras (Super, 1957), ao qual o nome de Super é mais popularmente associado, é aquele das abordagens de desenvolvimento. Como refere Bujold (1989) e Bujold e Gingras (2001) compilando as informações de Super (1984), esta concepção propõe que o desenvolvimento vocacional é um processo contínuo desde a infância até a velhice. O desenvolvimento é, geralmente, ordenado e previsível, assim como dinâmico no sentido de que ele resulta da interação entre as características do indivíduo e as demandas da cultura, o que torna claro também o fato de tratar-se de um processo psicossocial. No decorrer deste processo, o indivíduo deve cumprir um certo número de tarefas de desenvolvimento. A natureza precisa destas tarefas e a maneira 
pela qual ele as cumpre revela sua maturidade vocacional. Dentro desta ótica, pode-se dizer de um indivíduo, que ele é maduro na medida em que ele está pronto para tomar as decisões e para assumir os comportamentos característicos de seu estado de desenvolvimento vital (Super, 1990; Super \& cols., 1996). A Tabela 1 apresenta, dentro do quadro dos megaciclos (crescimento, exploração, estabelecimento, manutenção e desengajamento), a lista das tarefas de desenvolvimento, estados e sub-estados correspondentes. Como explica Super (1990), os estados têm uma tendência a se mesclar e não são, portanto, claramente definidos por limites de idade. Além disso, não somente as idades de transição são muito flexíveis, mas cada transição comporta uma reciclagem através de um ou mais estados, isto é, um miniciclo. Por exemplo, no estado megacíclico de desengajamento, o indivíduo deve pensar em desenvolver novos papéis não ocupacionais (crescimento), procurar um lugar apropriado onde se retirar (exploração), realizar atividades que ele sempre quis fazer (estabelecimento), continuar a fazer as atividades que ele sempre amou fazer (manutenção) e reduzir suas horas de trabalho (desengajamento).

4) O quarto modelo é denominado fenomenológico, e também de autoconceito - precisamente, sistemas de autoconceitos. Segundo Super (1990), o autoconceito, e mais precisamente o autoconceito profissional - que analisa os conjuntos de traços da pessoa diretamente ligados ao seu desenvolvimento profissional, sendo esses, seus interesses, seus valores e suas aptidões - tem um papel organizacional maior como guia do comportamento do indivíduo através dos estados e sub-estados do desenvolvimento vocacional. Desta forma, ele escreveu: “ ... formulando uma preferência vocacional, o indivíduo exprime uma idéia do tipo de pessoa que pensa ser; escolhendo uma profissão, ele atualiza seu autoconceito; progredindo numa carreira, ele se atualiza" (Super, 1963a, p. 4).

Como se pode constatar, é no terceiro modelo desta grande e integradora teoria que se agrega à noção de maturidade vocacional. Super (1955) inicialmente focalizou esta noção quando escreveu, pela primeira vez, sobre as dimensões e medidas da maturidade vocacional. Ele queria, assim, estabelecer os critérios para descrever e avaliar os estados e os sub-estados do desenvolvimento da carreira de estudantes com diferentes idades e níveis de estudos, assim como as tarefas do desenvolvimento com as quais eles estavam confrontados e seu nível de preparação para resolvê-las, para, finalmente, escolher uma carreira. Mais tarde (Super, 1963b), ele tentou definir esta noção de uma maneira operacional escrevendo de forma ainda mais detalhada as tarefas de desenvolvimento, assim como os atributos e comportamentos característicos da adolescência e da vida adulta, como pode-se encontrar na Tabela 1. Em seus trabalhos mais recentes (Super, 1990; Super \& cols., 1996), a maturidade vocacional é definida como a capacidade do indivíduo para enfrentar as tarefas de desenvolvimento com as quais ele é confrontado como conseqüência de seu desenvolvimento social e biológico, de uma parte, e das necessidades da sociedade em relação às outras pessoas que alcançam este estado de desenvolvimento, de outra parte.

É conveniente precisar, quando se fala de maturidade vocacional no modelo desenvolvimentista de Super, que esta noção se aplica propriamente ao desenvolvimento profissional do adolescente e do início da idade adulta. Com efeito, no caso dos adultos, Super (Super \& cols., 1996) não fala exatamente de maturidade vocacional, mas sim de adaptabilidade profissional. Esta distinção na conceitualização deve ser feita pela boa razão que, como é o caso para outros aspectos da personalidade, tal qual o desenvolvimento intelectual (Balbinotti, 1994), o desenvolvimento vocacional não está em relação linear com a idade cronológica (Balbinotti \& Tétreau, 2001a, 2001b, 2002). Portanto, esclarece-se que o significado é o mesmo, troca-se apenas os títulos dos conceitos.

Continua-se, então, respondendo a questão sobre maturidade vocacional centrando-se principalmente no desenvolvimento profissional dos adolescentes e de jovens adultos; quando necessário, tocar-se-á na noção de adaptabilidade profissional.

\section{Estudos de Verificação Empírica}

Super e colaboradores, no Teachers College da Universidade de Columbia, e mesmo diversos outros pesquisadores, já tentaram colocar à prova sua teoria de diversas maneiras, mas, a mais conhecida é, sem dúvida, aquela que representa seu estudo de padrões de carreira (Career Pattern Study - CPS), de 1985. Trata-se de um estudo longitudinal iniciado em $1951 \mathrm{com}$ jovens de $9^{\circ}$ ano escolar (entre 14 e 15 anos) e que durou os 25 anos seguintes, isto é, até o momento em que a maior parte dos sujeitos $(70 \%$ deles, precisamente), estavam estabilizados em um padrão de carreira. Este estudo permitiu estabelecer as relações entre a maneira pela qual os sujeitos passavam pelas tarefas de desenvolvimento profissional correspondentes ao estágio de exploração na adolescência (cristalização, 14-18 anos) e o status de seus comportamentos vocacionais no estágio de estabelecimento (estabilização, 21-30 anos e 30-45 anos em uma profissão), por comparação com as predições deste mesmo status a partir das medidas convencionais das características dos sujeitos (inteligência, rendimento escolar, nível socioeconômico e autoconceito vocacional).

Para melhor definir a natureza das relações, a tarefa da cristalização e o status do indivíduo na etapa do 
estabelecimento profissional, convém descrever, mais especificamente, o que Super entende como tarefa de cristalização de uma preferência profissional na adolescência.

Segundo Super (1963c), a formulação de uma preferência geral e a constância na sua expressão constituem os principais critérios da cristalização na adolescência. Para chegar lá, o adolescente deveria verdadeiramente estar, inicialmente, consciente da necessidade de cristalizar uma preferência de pensar, e, em seguida, considerar os fatores pessoais e ambientais pertinentes (aptidões, informações dos pais, dos conselheiros, etc...), notadamente as contingências econômicas podendo influenciar as realizações de seus objetivos vocacionais. Ele deveria, também, tornar-se consciente das relações entre suas atividades prévocacionais e suas escolhas vocacionais subseqüentes, assim como seu perfil de interesses e de valores; em verdade, quanto mais preciso for seu conhecimento dos interesses e dos valores, mais diferenciado será seu perfil, e mais simples será sua tarefa de cristalizar sua preferência. Ele deverá enfim, não somente estar consciente, mas também utilizar os recursos que o permitirão realizar suas atividades de planificação e de exploração de uma carreira. Este pano de fundo que Super assina, por assim dizer, ao adolescente, pode se resumir em termos de duas dimensões (planificação e exploração) da maturidade vocacional que os primeiros dados de seu estudo longitudinal, sobre o desenvolvimento dos padrões de carreira com os meninos de $9^{\circ}$ ano (Super \& Overstreet, 1960), permitiram identificar. Sendo assim, este estudo confirmou a hipótese que a expressão de escolhas específicas nesta idade é habitualmente irrealista ou prematura, e que o mais importante, no início da adolescência, é chamar a atenção dos jovens sobre os processos de decisão que subentendem a escolha da carreira, como a responsabilidade de elaborar planos de ação e utilizar os recursos ambientas disponíveis para exploração.

Os dados subseqüentes sobre a maturidade vocaciona destes mesmos meninos, quando estavam mais velhos, no $12^{\circ}$ ano escolar, permitiram a Jordaan e Heyde (1979) concluir, de acordo com a formulação teórica de Super, existência de uma certa progressão em relação àquela que prevalecia no $9^{\circ}$ ano escolar; isto é, podia-se notar uma maior cristalização e uma maior especificação da preferências, e que a eminência das escolhas a fazer tinha verdadeiramente influenciado (Perron, 1967). Eles também estavam mais confiantes, no $12^{\circ}$ ano de estudos, na validade de seus interesses que tinham se transformado, por assim dizer, estando agora, mais comparáveis aos dos adultos. Entretanto, se estas modificações tornaram-se mais perceptíveis quanto ao conjunto dos sujeitos, os índices de maturidade vocacional, no nível individual, não apresentavam um quadro assim tão claro. Certos meninos tinham feito progressos, alguns tinham estagnado e outros tinham perdido terreno. Estes resultados evidenciaram que no desenvolvimento vocacional, durante o ensino secundário, pode faltar constância e uniformidade. Coallier (1992), recentemente, confirmou esses resultados com uma amostra de adolescentes canadenses. Mais recentemente ainda, Balbinotti (2001) reconfirmou estes resultados, com uma amostra de 5200 adolescentes gaúchos.

Com o estudo do CPS, Super (1985b) pode concluir que as medidas de maturidade vocacional, no $12^{\circ}$ ano escolar (graus de planificação, de exploração e de preparação a tomar as decisões que requerem as necessidades sociais e as circunstâncias), são também mais potentes como preditores de variáveis critérios ao longo de 25 anos (status ocupacional esperado, avanço ocupacional, satisfação ocupacional, progresso e satisfação na carreira) do que as medidas convencionais (inteligência, status socioeconômico, sucesso escolar, etc.) e do autoconceito (precisamente duas metadimensões: harmonia e idiossincrasia). Como não se poderia duvidar, a utilização combinada de dois tipos de medida melhoram as predições, mas é conveniente remarcar que, já no $9^{\circ}$ ano escolar, certas características e comportamentos têm uma relação com o desenvolvimento de carreira e a atualização de uma preferência profissional 10 anos mais tarde, notadamente o status ocupacional esperado e o progresso na carreira.

Se o estudo do CPS permitiu, entre outros importantes resultados, mostrar a utilidade das medidas de maturidade vocacional como variáveis independentes para predizer o desenvolvimento vocacional subseqüente, outros autores (Jepsen, Dustin \& Miers, 1982; Schenk, 1979; Seyfarth, 1973; Ullery \& O'Brien, 1970) sublinharam a pertinência de se servir como variável dependente aos fins de identificar a eficácia de certos métodos de aconselhamento de carreira.

\section{A Medida da Maturidade Vocacional}

No entusiasmo dos primeiros resultados do CPS, rapidamente apareceu a idéia de que seria oportuno para os pesquisadores e os técnicos terem instrumentos válidos de avaliação da maturidade vocacional. Foi neste furor que se publicou, em 1972, a primeira versão do Career Development Inventory (CDI) (Forrest \& Thompson, 1974). Concebido para ser utilizado, basicamente, com adolescentes, esta primeira versão do CDI foi, em seguida, modificada, respondendo assim a melhores qualidades psicométricas e dando lugar às versões do CDI-A (para jovens adultos) e do Adult Career Concerns Inventory (ACCI) (Super, Thompson \& Lindeman, 1988). Teve-se a oportunidade de verificar que estes instrumentos foram, conseqüentemente, traduzidos ou adaptados com amostras 
de adolescentes e adultos de diversos países (Espanha, Canadá, França, Áustria, África do Sul, Países Baixos, Portugal, Reino Unido e Austrália). Estes e outros instrumentos, quando inspirados na teoria de Super, foram elaborados para se poder medir certas dimensões da maturidade vocacional. Refere-se precisamente ao Career Maturity Inventory (CMI) (Crites, 1978) e ao Questionário de Educação à Carreira (QEC) (Dupont \& Gingras, 1990), que, por um lado, Gonzalez (1992) assegurou a tradução e a adaptação na Espanha e, por outro lado, Balbinotti e Tétreau (2002) asseguraram as qualidades psicométricas para uso no Brasil. No mais, o Inventário de Desenvolvimento Profissional (IDP), do qual Dupont e Marceau (1982) demonstraram as qualidades psicométricas através da ajuda de dados colhidos pela aplicação deste em estudantes do ensino médio canadenses, de ambos os sexos, mede claramente as dimensões correlatas ao conceito de maturidade vocacional de Donald Super. Uma versão brasileira deste último foi assegurada por Lobato (2001).

Caminho feito, salienta-se que os 110 itens do CDI foram concebidos para avaliar os dois componentes gerais da maturidade vocacional: atitude e cognitivo. Ele permite calcular oito escores por meio de quatro escalas e da combinação destas: planificação (25 itens), exploração (25 itens), atitudes em relação à carreira e tomada de decisão (20 itens), informação sobre o mundo do trabalho (40 itens), conhecimento do processo de desenvolvimento da carreira, conhecimento do grupo ocupacional preferido, e escore total de orientação à carreira.

Quanto ao ACCI, a fim de identificar em qual etapa de seu caminho profissional o sujeito se percebe, os itens medem as preocupações com as tarefas de desenvolvimento da carreira segundo os estados e sub-estados que aparecem na Tabela 1. A Tabela 2 apresenta um resumo dos coeficientes de consistência interna (Alpha de Cronbach ou Fórmula KR-20) obtidos por diversos instrumentos de medida da maturidade vocacional e da adaptabilidade profissional (nos adultos).

\section{Perspectiva Transcultural da Noção de Maturidade} Vocacional

Super $(1957,1990)$ sempre considerou que os fatores socioeconômicos e ambientais, e aí se compreende os fatores culturais e de considerações étnico-raciais (precisamente o segundo modelo de sua teoria), podem influenciar o processo de desenvolvimento de uma carreira. Ele estima, com efeito, que esses fatores podem condicionar este processo de duas formas distintas. A primeira delas referese às oportunidades de desenvolvimento que eles facilitam ou dificultam, e a segunda, à procura de modelos ocupacionais e de autoconceitos. Segundo Fouad e Arbona (1994), mesmo Psicologia: Reflexão e Crítica, 2003, 16(3), pp. $461-473$ que a teoria de Super tenha sido elaborada a partir de amostras de homens brancos, nos Estados Unidos, ela seria generalizável a outras culturas e populações. Assim, diversos estudos foram realizados a fim de verificar se a teoria de Super é realmente válida quando se considera tais fatores.

\section{Pesquisas Americanas}

Com o CDI

O CDI foi desenvolvido nos Estados Unidos e suas qualidades psicométricas foram verificadas e confirmadas em diversos estudos e com amostras independentes (Dean, 1982; Kuhlman-Harrison \& Neely, 1980; Landro, 1983; Mello, 1983; Super, 1973, 1977; Super \& Thompson, 1979; Super \& Zelkowitz, 1974; Tilden, 1976; Westbrook \& Mastie, 1973). Feito este caminho, a maturidade vocacional, tal qual medida pelo CDI, também foi objeto de estudos e relações com outras variáveis.

Entre os estudos pioneiros do contexto socioeconômico e cultural com relação à maturidade vocacional, é conveniente destacar o trabalho de Vondracek, Lerner e Schulenberg (1986). Segundo estes autores, as atividades nas quais as crianças e os jovens adolescentes se engajam, a qualidade das relações interpessoais de seus contextos social e familiar, as experiências únicas ou idiossincráticas de suas existências, da mesma forma que os papéis que eles têm a oportunidade de observar e de se implicar, exercem uma grande influência sobre os seus desenvolvimentos vocacionais. As pesquisas empíricas repertoriadas neste trabalho sobre as relações da maturidade vocacional com diversas outras variáveis são apresentadas abaixo.

Em um estudo longitudinal da relação entre maturidade vocacional e sucesso acadêmico de 111 estudantes de medicina de uma universidade da Califórnia, de ambos os sexos, Lewis, Savickas e Jones (1996) constataram que o grau de maturidade vocacional é um potente preditor de sucesso. Eles também verificaram que esta variável pode ser mais potente que outras variáveis ligadas ao sucesso escolar, notadamente a inteligência e o nível socioeconômico. Em um estudo de sua relação com a percepção de barreiras ao avanço ocupacional com 188 colegiais do Texas, Luzzo (1996) concluiu a ausência de relações significativas ( $\mathrm{p}>0,05)$. Por outro lado, Kenny (1990) encontrou que a maturidade vocacional tem relação significativa com os valores do trabalho e a estrutura familiar. No que se refere ao estudo de Dykeman (1983), este constatou que três das quatro dimensões do modelo de maturidade vocacional de Super (planificação, exploração e tomada de decisão) são significativamente correlacionadas a eficiência no trabalho. No estudo de McCaffrey, Miller e Winston (1984), eles confirmaram, com a ajuda de uma amostra de 60 estudantes de nível superior, a hipótese já verificada com os adolescentes 
no estudo do CPS (Jordaan e Heyde, 1979) que, de maneira geral e não necessariamente linear, o nível de maturidade vocacional aumenta em função do de estudos.

A propósito dos estudos transculturais no interior dos Estados Unidos, Smallman e Sowa (1996) examinaram as respostas ao CDI de 125 estudantes (atletas de diversos esportes) de origens culturais diversas: caucasianos, africanos, asiáticos, hispânicos e de tradição indígena. Os resultados não apresentaram nenhuma diferença significativa quanto ao fator atitude (planificação e exploração). Entretanto, os sujeitos de origem caucasiana demonstraram um nível significativamente mais elevado de conhecimento das ocupações preferidas em relação às outras culturas. No mais Kelly e Cobb (1991) não encontraram diferença significativas nos graus de maturidade profissional de adolescentes superdotados de origem africana e caucasiana. Por outro lado, estes mesmos autores observaram uma diferença significativa entre os superdotados (de qualquer origem) e os "normais". Ainda, Henry, Bardo e Henry (1992) obtiveram resultados que sugerem que a média das respostas dos afro-americanos de sua amostra era mais baixa que aquela dos caucasianos. Outros estudos apresentaram resultados similares, isto é, sujeitos de origem africana geralmente obtêm escores menos elevados de maturidade vocacional que os caucasianos (Fouad \& Kelly, 1992 Westbrook \& Sanford, 1991). Desta forma, como sublinham Fouad e Arbona (1994), os resultados destes mesmos estudos confirmam a hipótese de Super, precisamente aquela da sucessão ordenada dos estados e das tarefas de desenvolvimento associadas. Dito de outra forma, assim como Leong (1993, 1995a, 1995b,), Leong e Brown (1995) e Leong e Hartung (1997) o fazem remarcar comparando a maturidade vocacional de estudantes caucasianos e asiáticos, se fatores culturais, como a menor importância acordada pelos asiáticos no que se refere ao valor do individualismo, por um lado, podem diminuir o estabelecimento de seus autoconceito e, por outro lado, podem tornar ainda mais complexas suas progressões através dos diferentes sub-estados de desenvolvimento profissional. A ordem destas progressões permanece, contudo, similar entre os dois grupos. Sem dúvida um achado favorável à compreensão multicultural da noção de maturidade enquanto processo de desenvolvimento, processo evolutivo.

$\mathrm{Na}$ medida que o fato de pertencer mais a um gênero (masculino ou feminino) pode ser concebida em termos de diferenças culturais, pode ser interessante notar que Thomason e Winer (1994) corroboraram os resultados de Super (1985a) no sentido que as meninas obtiveram escores de maturidade vocacional mais elevados que aqueles dos meninos. Dados semelhantes a estes foram encontrados no estudo de Balbinotti (2001) e Balbinotti e Tétreau (2002), a partir de duas amostras independentes de adolescentes sul-brasileiros com a ajuda do QEC.

\section{Com o ACCI}

Como no caso do CDI, o ACCI foi elaborado nos EUA. Diversos autores (Halpin, Ralph \& Halpin, 1989; Mahoney, 1987; McCloskey, 1986; Savickas, Passen \& Jarjoura, 1988; verificaram e confirmaram suas qualidades psicométricas e outros (Halpin, Ralph \& Halpin, 1990; Super, 1983; Super, Osborne, Walsh, Brown \& Niles, 1992; Whiston, 1990) sublinharam a pertinência de sua utilização no plano prático. Neste sentido, Niles e Anderson (1993) buscaram verificar a relação entre o stress e a adaptabilidade profissional utilizando uma amostra de 110 clientes onde 76 eram mulheres; seus resultados sugeriram que os homens que manifestavam pouca adaptabilidade profissional experimentavam um nível mais elevado de stress ocupacional. Mais recentemente Booth (1994) reexaminou a estrutura fatorial do ACCI lançando mão de equações estruturais (confirmatory factor analisis) e chegou a conclusão que se trata de uma medida também aplicável a pessoas deficientes. Ralph (1986) e Ralph (1987) afirmam que este instrumento foi utilizado em alguns estudos em relação a outros instrumentos a fim de se verificar a validade conceitual. Ralph (1987) o administrou em 109 sujeitos adultos e seus resultados mostraram que a adaptabilidade profissional está em correlação positiva e significativa com a autoconfiança.

\section{Pesquisas em Outros Países \\ Com o CDI}

Versões adaptadas e normatizadas deste inventário foram utilizadas com sucesso em outros países, notadamente no Canadá (Dupont \& Marceau, 1982), na Espanha (Moreno, 1987), na Áustria (Seifert \& Eder, 1985), na África do Sul (Watson, Stead \& Jager, 1995), na Suíça (Descombes, Frischknecht, Dupont \& Huter, 1977), em Portugal (Ferreira \& Caeiro, 1981), na Austrália (Punch \& Sheridan, 1985) e na Inglaterra (Ward, 1982). Entretanto, os resultados obtidos nestes estudos, os quais foram favoráveis, não parecem ter tido continência no caso do Brasil (Fernandes \& Scheeffer, 1986; Scheeffer \& Fernandes, 1984) e nos Países Baixos (Helbing, 1984) onde os resultados não puderam permitir conclusões positivas quanto a sua utilização na população adolescente. As estruturas fatoriais obtidas através de análises multidimensionais demonstraram a necessidade da retirada de diversos itens para que o instrumento pudesse conservar uma estrutura, no mínimo, aceitável.

No Canadá, Young (1984) examinou a relação entre as aspirações ocupacionais e a maturidade vocacional de uma amostra de 590 adolescentes de seis escolas rurais. Ele 
encontrou que, quando estes jovens eram comparados aos grupos de adolescentes que tinham escolhido ocupações tradicionais, os adolescentes que aspiravam ter sucesso em ocupações inovadoras manifestavam um grau de maturidade significativamente mais elevado. Considerando a elaboração de uma estratégia de avaliação de necessidade de educação a carreira de 1022 estudantes canadenses franceses de ambos os sexos e todos do último ano do ensino médio no Quebéc, Gingras (1991) utilizou o QEC. Ela observou que os estudantes tinham se comprometido muito pouco em suas atividades de planificação e de exploração de uma possível carreira futura, não aproveitando grande parte das fontes de ajuda e informações que estavam propostas neste instrumento. Gonzalez (1992), na Espanha, obteve resultados similares com a ajuda de uma amostra de 2997 adolescentes emparelhados com o CEC, a versão Espanhola do QEC, podendo se tirar as mesmas conclusões.

$\mathrm{Na}$ Áustria, Seifert (1985) administrou a versão austríaca do CDI em uma amostra de 641 adolescentes de oitavo e nono anos escolares. Os resultados sugerem que estes adolescentes manifestaram pouca maturidade vocacional e que os conselheiros e psicólogos escolares deveriam facilitar as informações vocacionais destes jovens, aumentando, em última análise, o nível desta informação. Em um outro estudo, seis anos mais tarde, Seifert (1991) explorou a relação entre a maturidade vocacional e os comportamentos associados às escolhas vocacionais de 1336 estudantes do $11^{0}$ e $12^{0}$ anos de estudos, final do ensino médio. Os resultados mostraram que os escores elevados de maturidade vocacional estavam significativamente associados às preocupações quanto ao objeto de escolha de carreira e ao sentimento de confiança de poder realizar seu projeto profissional.

Watson e colaboradores (1995) realizaram um estudo na África do Sul, com uma amostra de 264 estudantes universitários, brancos e pretos, a fim de verificar a relação do gênero e da cultura com a maturidade vocacional. Os resultados demonstraram que não existem diferenças significativas entre a maturidade e o gênero, nesta amostra. Entretanto, os estudantes brancos obtiveram escores de maturidade significativamente mais elevados que os estudantes negros. Contudo, os autores sugerem prudência quanto às possíveis conclusões que podem ser interpretadas a partir destes resultados. Antes de qualquer interpretação precipitada, os autores sublinham a necessidade de um aprofundamento maior e salientam que os estudantes negros de sua amostra habitavam em um bairro que sofreu muitos problemas de instabilidade política e social, naquele país. Assim, a sociedade sul-africana em geral, é ainda objeto de muita discriminação tanto na estrutura política quanto nas estruturas social, econômica e escolar. Desta forma, tais variáveis podem constituir um viés importante tanto em termos de medida quanto de interpretação desses resultados.

Um outro estudo foi realizado com quatro estudantes de escola média, no México (utilizando entrevistas semiestruturadas em uma pesquisa de caráter qualitativo). Neste estudo, Bullington e Arbona (1991) corroboram os avanços teóricos de Super observando que estes quatro estudantes estavam implicados nas tarefas exploratórias de planificação e exploração, de acordo com seus estados de vida e, portanto, de acordo com a sistematização teórica de Super.

Fouad (1988) comparou a maturidade vocacional de 875 estudantes americanos de nível médio, de nono e décimo segundo ano de estudo, com 537 estudantes israelenses dos mesmos níveis. Os resultados demonstraram diferenças significativas entre as duas culturas assim como entre o sexo e o nível de estudos (entre os israelenses). De modo geral, os estudantes israelenses obtiveram escores de maturidade vocacional significativamente menos elevados que aqueles obtidos por estudantes americanos. Contrariamente a este último resultado, os israelenses se mostraram mais decididos, quanto a seus projetos de carreira, revelando assim uma maior capacidade na execução de compromissos entre a realidade e suas próprias necessidades. A autora conclui que as tarefas do desenvolvimento de estudantes em diferentes culturas são similares. Entretanto, o tempo necessário para atingir o sucesso pode variar segundo a cultura e os inventários de maturidade podem não estar ainda adaptados a estas diferenças.

Comparando estudantes americanos e árabes, de nono e décimo segundo ano escolar, no Líbano, Moracco (1976), verificou que os primeiros tiveram escores mais elevados que os segundos. Conforme sua opinião, esta diferença seria atribuível a fatores culturais.

\section{Com o ACCI}

Os resultados das análises fatoriais do ACCI ou de suas traduções adaptadas com adultos desempregados ou em formação profissional, ou ainda com adultos trabalhadores $(n=457)$ na Austrália (Smart \& Peterson, 1994), em Portugal $(n=881)$ (Duarte, 1995), no Canadá $(n=1554)$ (Dupont, Gingras \& Tetreau, 1992a, 1992b) e na França $(n=1414)$ (Gelpe, 1997) confirmaram a presença dos quatro fatores constitutivos de sua estrutura fatorial da versão original (exploração, estabelecimento, manutenção e desengajamento). Os índices obtidos a partir de cálculos de consistência interna são também satisfatórios. O ACCI é um instrumento ainda relativamente novo de sorte que ele ainda não foi extensivamente utilizado pelos práticos. 


\section{Conclusão}

Depois do início dos anos 1940, ainda no século passado, Super engajou-se a sistematizar, de maneira exaustiva, uma teoria geral da escolha e do desenvolvimento vocacional. Esta teoria é estruturada em quatro modelos distintos, mas conexos: o modelo tradicional, o modelo dos fatore socioeconômicos e ambientais, o modelo das abordagens desenvolvimentistas e, finalmente, o modelo fenomenológico. O presente trabalho objetivou não somente a compreensão e a apresentação do conceito de maturidade vocacional, mas igualmente sua gestão no plano transcultural. Com este propósito, explorou-se, mais especificamente, o terceiro modelo que aborda a dinâmica desenvolvimentist do cumprimento de etapas ordenadas e previsíveis durante toda a vida do indivíduo, sublinhando as tarefas inerentes ao desenvolvimento vocacional. A noção de maturidade vocacional se revela através da passagem deste processo. Esta noção foi recentemente definid (Super \& cols., 1996) como a capacidade do indivíduo tomar decisões e assumir os comportamento característicos de seu estado (ver Tabela 1). Dentro de uma ótica que privilegia a compreensão pormenorizada desta dinâmica de desenvolvimento, Super (1985a, 1985b) conduziu um estudo longitudinal, o CPS, no qual a noção de maturidade vocacional tinha um papel organizaciona de destaque. Depois desta pesquisa fundamental pioneira, diversos autores, em diferentes países e/ou culturas, investiram em várias pesquisas empíricas a fim de explorar, sistematicamente, o conceito de maturidade vocacional e sua relação com outras variáveis tais como o sucesso escolar, os valores do trabalho, as aspirações e os interesses profissionais, entre outras.

Diversos instrumentos, inspirados a partir da teoria do desenvolvimento vocacional de Super, foram criado (CDI, CMI, QEC, IDP, ACCI). No que se refere às pesquisas trans-culturais com o CDI e o ACCI, notadamente, pode-se concluir que a estrutura fatorial destes instrumentos pode ser encontrada, na maioria de suas versões traduzidas e/ou adaptadas em diversos países. Encontrou-se então, essencialmente, as quatro grandes etapas (os megacíclos) no que concerne o ACCI e o dois fatores (atitude e cognitivo) no que concerne o CDI Observa-se (ver Tabela 2) também que os coeficientes de consistência interna são muito similares (geralmente elevados ou muito elevados) entre um país e outro. A única exceção seriam os dados obtidos com o CMI, no Brasil, por Japur (1988) e Japur e Jacquemin (1989). Assim, o processo ordenado de desenvolvimento vocacional seria então, em grande parte, generalizável no plano universal, ao menos para os países estudados. Entretanto, diferenças relativamente importantes são observadas segundo as amostras utilizadas, isto é, se elas são constituídas de homens ou de mulheres, de sujeitos com mais ou menos idade, com sujeitos pertencentes a uma maioria branca ou uma minoria visível, com sujeitos que habitam em bairros mais ou menos favorecidos, ou ainda se eles pertencem a um ou outro grupo étnico. Parece importante considerar todos estes aspectos no momento de interpretar os resultados originários dos levantamentos de dados sobre maturidade vocacional.

Considerando as diferenças segundo o pertencimento racial ou étnico, pode-se perguntar, concluindo, se não se trata, antes de tudo, de diferenças de nível socioeconômico e de valores associados a elas. Por exemplo, sabe-se que os indivíduos desfavorecidos no nível socioeconômico podem valorizar mais dificilmente as gratificações diversas (não em dinheiro) de uma forma diferenciada dos indivíduos de nível socioeconômico mais elevado. Podese então formular a hipótese de que os indivíduos com um nível socioeconômico alto obterão médias significativamente mais elevadas no estado do Estabelecimento, medido pelo ACCI, do que aqueles de nível socioeconômico mais baixo. Pois, tendo tido, mais rapidamente, acesso ao mercado de trabalho eles se estabilizarão também mais rapidamente em um padrão de carreira. Pode-se pensar, por exemplo, no caso de um jovem que se torna policial na idade de 18-19 ou 20 anos, e planeja fazer carreira nesta profissão na qual ele se estabelecerá relativamente cedo, por oposição ao caso de um estudante de graduação em psicologia que se pergunta se ele será admitido em estudos de pósgraduação ou se ele deverá remeter em questão sua orientação nesta via e recomeçar a explorar uma outra abordagem diferente da psicologia.

O modelo parece então generalizável a diversas populações que possuem um mínimo de elementos culturais comparáveis aos americanos e, mais geralmente, ao mundo ocidental. Uma questão que se pode levantar é se o modelo é generalizável a uma ou outra amostra em particular. Pode-se supor que as médias das amostras vão provavelmente ser diferentes segundo o nível socioeconômico, conforme a importância acordada ao trabalho e ao seu papel na vida do indivíduo, de acordo com o tipo de trabalho e a carreira a que está associada (estabelece-se muito cedo em uma profissão de atleta profissional, e relativamente tarde naquela de juiz), e com relação à real possibilidade ou não de trabalhar. 
Tabela 1

Relação dos Estados e Sub-estados do Ciclo Vital com as Tarefas de Desenvolvimento

\begin{tabular}{|c|c|}
\hline Estados e sub-estados & Tarefas de desenvolvimento \\
\hline \multicolumn{2}{|l|}{ Crescimento (0-14 anos) } \\
\hline $\begin{array}{l}\text { - Sub-estado fantasista e de curiosidade } \\
\text { - Sub-estado do desenvolvimento dos interesses e das } \\
\text { aptidões }\end{array}$ & $\begin{array}{l}\text { - Aprendizagem das técnicas gerais de adaptação e de } \\
\text { formação de um autoconceito vocacional. }\end{array}$ \\
\hline \multicolumn{2}{|l|}{ Exploração (14-25 anos) } \\
\hline - Sub-estado provisório & - Cristalização de uma preferência. \\
\hline - Sub-estado de transição & - Especificação de uma preferência. \\
\hline - Sub-estado de tentativas (pouco engajamento) & - Atualização de uma preferência. \\
\hline \multicolumn{2}{|l|}{ Estabelecimento (21-45 anos) } \\
\hline - Sub-estado de tentativa e estabelecimento & - Estabilização em uma profissão. \\
\hline - Sub-estado de avanço & - Consolidação do status e avanço. \\
\hline \multicolumn{2}{|l|}{ Manutenção (40-65 anos) } \\
\hline - Sub-estado de adaptação e preservação do autoconceito & - Manter posição, atualizar-se e inovar. \\
\hline \multicolumn{2}{|l|}{ Desengajamento (65 anos e mais) } \\
\hline $\begin{array}{l}\text { - Sub-estado de adaptação a um novo selfe ao processo } \\
\text { de aposentadoria }\end{array}$ & $\begin{array}{l}\text { - Desaceleração, planificação da aposentadoria e da vida } \\
\text { de aposentado. }\end{array}$ \\
\hline
\end{tabular}

Tabela 2

Consistência Interna de alguns Instrumentos de Medida da Maturidade Vocacional e da Adaptabilidade Profissional, Considerando os Diversos Países

\begin{tabular}{ccc}
\hline Países & Instrumentos & Alpha \\
\hline Canadá & QEC & $0,66-0,93$ \\
Canadá & IDP & $0,64-0,88$ \\
Canadá & IPC & $0,77-0,95$ \\
Estados Unidos & CMI & $0,65-0,90$ \\
Estados Unidos & CDI & $0,61-0,91$ \\
Estados Unidos & CDI-A & $0,92-0,95$ \\
Estados Unidos & ACCI & $0,76-0,95$ \\
França & IPP & $0,81-0,93$ \\
Espanha & CEC & $0,71-0,94$ \\
Portugal & ACCI & $0,83-0,97$ \\
Brasil & QEC & $0,73-0,93$ \\
Brasil & IDP & $0,36-0,91$ \\
Brasil & CMI & $0,32-0,67$ \\
\hline
\end{tabular}

Psicologia: Reflexão e Crítica, 2003, 16(3), pp.461-473 


\section{Referências}

Balbinotti, M. A. A. (1994). Significado social do conceito e da avaliacão da inteligênciz: Perspectiva histórica e levantamento de opiniōes. Dissertação de Mestrado nãopublicada, Programa de Pós-Graduação em Psicologia Social e da Personalidade, Faculdade de Psicologia, Pontifícia Universidade Católica do Rio Grande do Sul. Porto Alegre, RS,

Balbinotti, M. A. A. (2001). Vers un modèle explicatif de la cristallisation des préferences professionnelles durant l'adolescence. Tese de Doutorado não-publicada, Programa de Pós-Graduaç̃oo em Psicologia, Université de Montréal. Montreal Quebec, Canadá.

Balbinotti, M. A. A. \& Tétreau, B. (2001a).Qualidadespsicométricas da versão brasileir do questionário de motivacão à carreira. (Material não-publicado)

Balbinotti, M. A. A. \& Tétreau, B. (2001b). Modelo explicativo da cristalivação das preferencias proffissionais (Manuscrito não-publicado, Université de Montréal) Balbinotti, M. A. A. \& Tétreau, B. (2002). L'Éducation à la Carrière : Une Étud Brésilienne de la Validité Transcuturelle du Questionnaire sur l'Éducation la Carrière. International Journal for Educational and Vocational Guidance, 2, 101 114.

Bingham, W. C. (1997). Technology, social isolation, and career development. Educational and Vocational Guidance Bulletin, 60, 46-56.

Bergeron, M. (1998). Le cyberespace et la relation patient-médicin. Interface, 19 $37-40$.

Blau, G. (1993). Work adjustment theory: A critique and suggestions for future research and application. Journal of Vocational Behavior, 43, 105-112.

Booth, W. D. (1994). Confirmatory factor analysis of the adult career concerns inventory in vocational rehabilitation clients: Towards a modal of caree development for people with disability. Dissertation Abstracts Internationatsection B: The Sciences \& Engineering, 54(8-B), 4428.

Breton, R., McDonald, J. \& Richer, S. (1972). Le rôle de l'école et de la société dans choix d'une carrière chez la jeunesse canadienne. Ottawa: Information Canada.

Brooks, L. (1984). Counseling special groups: Women and ethnic minorities. Em D. Brown, L. Brooks \& cols. (Orgs.), Career choice and development (pp. 355-368). San Francisco: Jossey-Bass.

Brown, M. T., Fukunaga, C., Umemoto, D. \& Wicker, L. (1996). Annual reiwew, 1990-1996: Social class, work, and retirement bahavior. Journal of Vocational Behavior, 49, 159-189.

Buehler, C. (1933). Der menschlicb lebenslauf als psychologisches problem [The human life course as a psychological subject]. Leipzig, GE: Hirzel.

Bujold, C. (1989). Choix professionnel et developpement de carrière. Boucherville, $\mathrm{PQ}$ Gaëtan Morin.

Bujold, C. \& Gingras, M. (2001). Choix professionnel et développement de carrière. Théories et recherches. Boucherville, PQ: Gaëtan Morin.

Bullington, R. L. \& Arbona, C. (1991). An exploration of the career development taskes of Mexican American youth. (Material não-publicado)

Coallier, J. -C. (1992). Étude des déterminants de la maturité vocationnelle dans une perspective multidimensionnelle chez deséềves de niveau secondaire. Tese de Doutorado não-publicada, Faculté des Arts et des Sciences, Université de Montréal. Montreal, Quebéc, Canada.

Crites, J. O. (1978). Career maturity inventory. Monterey, CA: CTB/McGraw-Hill.

Dean, S. A. (1982). A construct validity study of the career development inventory and the attitude scale of the career maturity inventory. Dissertation Abstracts International, 42(10-A), 4298.

Descombes, J.P., Frischknecht, E., Dupont, J. B. \& Huter, M. (1977). Occupational maturity. International Review of Applied Psychology, 26, 115-125.

Duarte, M. E. (1995). Career concerns, values, and role salience in employed men. Career Development Ouarterly, 43, 338-349.

Dupont, P. \& Gingras, M. (1990). Questionnaire sur l'éducation à la carrière. Sherbrooke, Université de Sherbrooke: Centre de recherche sur l'éducation à la carrière et le travail.
Dupont, P., Gingras, M. \& Tétreau, B. (1992a). L'Imventaire des préoccupations de carrière, pour adulte. Centre de recherche sur l'éducation et le travail, Université de Sherbrooke.

Dupont, P., Gingras, M. \& Tétreau, B. (1992b). A French Canadian adaptation of the Adult Career Concerns Inventory (A.C.C.I.). XXIV Congrès International de Psychologie, Bruxelles.

Dupont, P. \& Marceau, D. (1982). Manuel de linvventaire de Développement Professionnel. Adaptation et normalisation. Sherbrooke: Université de Sherbrooke.

Dykeman, B. F. (1983). Correlation of vocational maturity and components of vocational maturity with rated work effectiveness. Education, $t$

Farmer, H. S. (1984). A shiny fresh minted penny. The Counseling Psychologist, 12, 141-144.

Farmer, H. S. (1997). Women's motivation related to mastery, career salience, and career aspiration: A multivariate model focusing on the effects of sex role socialisation. Journal of Career Assessment, 5, 355-382.

Fernandes, L. M. \& Scheeffer, R. (1986). Construction of the Brazilian Instrument of Vocational Maturity. Arquivos Brasileiros de Psicologia, 38, 186-197.

Ferreira, M. J. H. \& Caeiro, L. A. (1981). The career development inventory in Portugal: A preliminary study. International Review of Applied Psychology, 30, 479-490.

Forrest, D. J. \& Thompson, A. S. (1974). The career development inventory. Em D. E. Super \& Cols. (Orgs.), Measuring vocational maturity for counseling and evaluation (pp. 53-66). Washington, DC: National Vocational Guidance.

Fouad, N. A. (1988). The construct of career maturity in the United States and Israel. Journal of Vocational Behavior, 32, 49-59.

Fouad, N. A. (1994). Annual review: 1991-1993: Vocational choice, decisionmaking, assessment and intervention. Journal of Vocational Behavior, 45, 125176

Fouad, N. A. \& Arbona, C. (1994). Careers in a cultural context. The Career Development Quarterly, 43, 97-112.

Fouad, N. A. \& Kelly, T. J. (1992). The relationship between attitudinal and behavioral aspects of career maturity. The Career Development Quarterly, 40, 257-271.

Gelpe, D. (1997). Les préoccupations de carrière d'adultes en situation de transition professionnelle: Effets de trois types de déterminants. L'Orientation Scolaire et Professionnelle, 26, 137-156.

Gingras, M. (1991). Élaboration d'une stratégie d'évaluation des besoins d'éducation à la carrière cher les finissants du secondaire. Tese de Doutorado não-publicada, Programa de Pós-Graduação em Psicologia, Université de Montréal. Montreal, Quebec, Canadá.

Ginzberg, E., Ginsburg, S. W., Axelrad, S. \& Herma, J. L. (1951). Occupational choice, an approach to a general theory. New York, NY: Columbia University Press.

Gonzalez, M. P. (1992). Les intervenciones educativas para el desarollo de la carrera: Analises de las necessidades de desarollo para la carrera de los estudiantes al finalizar la educcacion secundária. Tese de Doutorado não-publicada, Universidad de Oviedo. Oviedo, Espagne.

Halpin, G., Ralph, J. \& Halpin, G. (1989, janeiro). Factor structure of the Adult Career Concerns Inventory: Validity and reliability. Comunicação apresentada na reunião anual da Mid South Educational Research Association, Little Rock, AR.

Halpin, G., Ralph, J. \& Halpin, G. (1990). The adult career concerns inventory: Validity and reliability. Measurement 2 Evaluation in Counseling \& Development, 22, 196-202.

Havighurst, R. J. (1952). Developmental tasss and education ( $2^{\mathrm{a}}$ ed.). New York: David McKay.

Havighurst, R. J. (1964). Youth in exploration and man emergent. Em H. Borow (Orgs.), Man in a world at work (pp. 215-236). Boston: Houghton Mifflin.

Havighurst, R. J. (1972). Developmental tasks and education ( $3^{\mathrm{a}} \mathrm{ed}$.). New York: David McKay.

Psicologia: Reflexão e Crítica, 2003, 16(3), pp.461-473 
Helbing, J. C. (1984). Vocational maturity, self-concepts and identity. International Review of Applied Psychology, 33, 335-350.

Henry, P., Bardo, H. R. \& Henry, C. A. (1992). The effectiveness of career development seminars on African American premedical students: A program evaluation using the Medical Career Development Inventory. Journal of Multicultural Counseling \& Development, 20, 99-112.

Herr, E. L. \& Lear, P. B. (1984). The family as an influence on career development Family Therapy Collections, 10, 1-15.

Holland, J. L. (1959). A theory of vocational choice. Journal of Counseling Psychology, 6,35-45.

Holland, J. L. (1973). Making vocational cboices. Englewood Cliffs, NJ: PrenticeHall.

Holland, J. L. (1985). Manual for the Vocational Preference Inventory. Odessa, FL: Psychological Assessment Resources.

Holland, J. L. (1992). Making vocational choices: A theory of vocational personalities and work environments $\left(2^{a}\right.$ ed.). Odessa, FL: Psychological Assessment Resources.

Holland, J. L. (1996). Exploring careers with a typology. American Psychologist, 51 397-406.

Holland, J. L. (1997). Making vocational choices: A theoy of vocational personalities and work environments ( $3^{\mathrm{a}}$ ed.). Odessa, FL: Psychological Assessment Resources.

Hotchkiss, L. \& Borow, H. (1996). Sociological perspective on work and career development. Em D. Brown, L. Brooks \& Cols. (Orgs.), Career choice and development ( $3^{\mathrm{a}}$ ed.) (pp. 281-334). San Franscisco: Josseuy- Bass.

Japur, M. (1988). Estudo sobre as qualidades psicométricas do Formulário de Aconselhamento (B-1) da Escala de Atitudes do Career Maturity Inventory (CMI) de J. O. Crites. Tese de Doutorado não-publicada, Curso de Pós-Graduação/Doutorado em Psicologia Clínica, Universidade de São Paulo. São Paulo, SP.

Japur, M. \& Jacquemin, A. (1989). Escala de Atitudes (B-1) do Inventário de Maturidade Profissional (CMI): 1. Análise psicométrica. Psicologia: Teoria e Pesquisa, 5, 297-314.

Jepsen, D. A., Dustin, R. E. \& Miers, R. (1982). The effects of problem solving training on adolescents career exploration and career decision making. Personal $\lesssim$ Guidance Journal, 61, 149-153.

Jordaan, J. P. \& Heyde, M. B. (1979). Vocational maturity during the High School years. New York: Teachers College Press

Kelly, K. R. \& Cobb, S. (1991). A profile of the career development characteristics of young gifted adolescents: Examining gender and multicultural differences. Roeper Reviem, 13, 202-206.

Kenny, M. E. (1990). College senior's perceptions of parental attachments: The value and stability of family ties. Journal of College Student Development, 31 $39-46$.

Knefelkamp, L. L. \& Slepitza, R. (1976). A cognitive-development model of career development: An adaptation of the Perry scheme. The Counseling Psychologist, 6, 53-58

Kuhlman-Harrison, J. \& Neely, M. A. (1980). Discriminant validity of career development scales in grade 10 students. Educational \& Psychological Measurements, 40, 475-478.

Landro, G. (1983). A validation study of the Career Development Inventory. Dissertation Abstracts International, 43(11-A), 3544-3545.

Lapointe, P. (1998). La complainte de linternaute. Interface, 19, 41-45.

Levinson, D. J., Darrow, C. N., Klein, E. B., Levinson, M. H. \& McKee, B. (1976), Periods in the adult development of men: Age 18 to 45 . The Counseling Psychologist, 6, 21-25.

Levinson, D. J., Darrow, C. N., Klein, E. B., Levinson, M. H. \& McKee, B. (1978). The seasons of a man's life. New York: Alfred A. Knopf.

Lewis, D. M., Savicks, M. \& Jones, B. J. (1996). Career development predicts medical school success. Journal of Vocational Behavior, 49, 86-98.

Leong, F. T. L. (1993). The career counseling process with racial-ethnic minorities: The case of asian americans. The Career Development Quarterly, 42, 26-40.

Psicologia: Reflexão e Crítica, 2003, 16(3), pp. $461-473$
Leong, F. T. L. (1995a). Career development and vocational behavior or racial and etbnic minorities. Mahwah, New Jersey: Erlbaum.

Leong, F. T. L. (1995b). Introduction and overview. Em F. T. L. Leong (Org.), Career development and vocational behavior or racial and etbnic minorities. Mahwah, New Jersey: Erlbaum.

Leong, F. T. L. \& Brown, M. T. (1995). Theoretical issues in cross-culturally career development: Cultural validity and cultural specificity. Em W. B. Walsh \& S. H. Osipow (Orgs.), Handbook of vocational psychology: Theory, research, and practice (pp. 143-180) (2 ed). Mahwah, NJ: Erlbaum.

Leong, F. T. L. \& Hartung, P. (1997). Career assessment with culturally different clients: Proposing and integrative-sequential conceptual framework for crosscultural career counseling research and practice. Journal of Career Assessment, 5 , 183-202.

Lobato, C. R. P. S. (2001). Maturidade vocacionale gênero: Adaptacão e uso de instrumentos de avaliacãa. Dissertação de Mestrado não-publicada, Programa de Pósgraduação em Psicologia do Desenvolvimento, Universidade Federal do Rio Grande do Sul. Porto Alegre, RS.

Luzzo, D. A. (1996). Exploring the relationship between the perception of occupational barriers and career development. Journal of Career Development, 22, 239-248.

Mahoney, D. J. (1987). An Exploration of the construct validity of a measure of adult vocational maturity. Dissertations Abstracts International, 47(11-A), 3983.

McCaffrey, S. S, Miller, T. K. \& Winston, R. B. (1984), Comparison of career maturity among graduates students and undergraduates. Journal of College Student Personnel, 25, 127-132.

McCloskey, G. M. (1986). An investigation of the content and construct validity of the adult career concerns inventory. Dissertation Abstracts International, $86(8-B), 2788$.

Mello, M. H. (1983). A critical analysis and evaluation of selected psychometric characteristics of the Career Development Inventory (School Form). Dissertation Abstract Inventory, 43(8-A), 2557-2558.

Miller, D. C. \& Form, W. H. (1951). Industrial sociology. New York: Harper \& Brothers.

Miller, J. V. \& Weeks, C. A. (1985). Family-career linkage. Journal of Carreer Development 12,1-89.

Moracco, J. C. (1976). Vocational maturity of arab and american high school students. Journal of Vocational Behavior, 8, 367-373.

Moreno, M. M. (1987). Evaluation of effectiveness of a vocational orientation intervention program. Revista de Analisis del Comportamiento, 3, 207-215.

Niles, S. G. \& Anderson, W. P. (1993). Career development and adjustment: The relation between concerns and stress. Journal of Employment Counseling, 30, 79-87.

Osipow, S. H. \& Fitzgerald, L. F. (1996). Theories of career development ( $4^{a}$ ed.). Boston: Allyn and Bacon.

Parsons, F. (1909). Choosing a vocation. Boston: Houghton Mifflin.

Perron, J. (1967). Quelques facteurs de décision vocationnelle en fonction de limminence du choix à faire. Problèmes d'orientation (Réjean et Jacques Perron) (pp. 31-38). Montréal: Corporation de psychologues de la Province du Québec.

Punch, K. F. \& Sheridan, B. E. (1985). Some measurement characteristics of the Career Development Inventory. Measurement e Evaluation in Counseling e Development, 17, 196-202.

Ralph, J. R. (1986). An investigation of self-concept, locus of control, and age as correlates of career maturity among graduate nursing students. Dissertation Abstracts International, 47(5-A), Nov. 1986, 1609, US: Univ. Microfilms International.

Ralph, J. A. (1987). Personality and demographic variables related to career development and career concerns. Auburn University. (Material não-publicado)

Riverin-Simard, D. (1977). Éducation permanente: Identification des stades du développement de l'adulte. L'orientation professionnelle, 13, 41-44. 
Riverin-Simard, D. (1981). Developement vocatinnel de l'adulte. L'orientation Professionnelle, 17, 7-32.

Riverin-Simard, D. (1984). Etapes de vie au travail. Montréal: Saint-Martin. Riverin-Simard, D. (1990). Carrières et classes sociales. Montréal: Saint-Martin. Riverin-Simard, D. (1993). Transitions professionnelles: Choixet stratégies. SainteFoy, Québec: Les Presses de l'Université Laval.

Riverin-Simard, D. (1996a). L'adulte au travail : Les variations de sa trajectoire. L'orientation Professionnelle, 9, 19-21.

Riverin-Simard, D. (1996b). Le concept du chaos vocationnel: Un pas théorique à l'aube du XXIe siècle? L'orientation Scolaire et Professionnelle, 25, 467-487.

Riverin-Simard, D. (1996c). Travailetpersonnalité. Sainte-Foy, Québec : Les Presses de l'Université Laval.

Riverin-Simard, D. (1998). Roles clés dans la révolution du travail. Sainte-Foy, Québec Les Presses de l'Université Laval.

Roe, A. (1956). The psychology of occupations. New York: Wiley.

Roe, A. (1957). Early determinants of vocational choice. Journal of Counseling Psychology, 4, 212-217.

Roe, A. \& Lunneborg, P. W. (1990). Personality development and career choice. Em D. Brown, L. Brooks \& cols. (Orgs.), Career choice and development: applying contemporary theories to practice (pp. 31-54) (2a ed). San Francisco: Jossey-Bass.

Savickas, M. L., Passen, A. J. \& Jarjoura, D. G. (1988). Career concern and copin as indicators of adult vocational development. Journal of Vocational Behavior, $33,82-98$.

Scheeffer, R. \& Fernandes, L. M. (1984). Factorial validity of the Brazilian Vocational Maturity Inventory. Arquivos Brasileiros de Psicologia, 36, 121-127.

Schenk, G. E. (1979). The influence of a Career group experience on the vocationa maturity of college students. Journal of Vocational Behavior, 14, 284-296.

Schultz, J. B. \& Henderson, C. (1985). Family satisfaction and job performance Implications for career development. Journal of Career Development, 1, 33-47. Seifert, K. H. (1985). Evaluation of guidance and counseling in Austria. International Journal for the Advancement of Counseling, 8, 55-74.

Seifert, K. H. (1991). Measures of career development and career choice behavior. Applied Psychology: An International Review, 40, 245-267.

Seifert, K. H. \& Eder, F. (1985). The career development inventory: The German adaptation. Zeiitschirft fur Differentielle und Diagnostische Psychologie, 6, 65-77.

Seyfarth, J. T. (1973). Analysis of scores on the CDI; employer based career education. Em Appalachia Educational lab, Charleston, W. V. Technical report no. 37. Smallman, E. \& Sowa, C. J. (1996). Career maturity levels of male intercollegiate varsity athletes. Career Development Quarterly, 44, 270-278.

Smart, R. \& Peterson, C. C. (1994). Super's stage and the four-factor structure of the Adult career concerns inventory in an Australian sample. Measurement e Evaluation in Counseling \& Development, 26, 243-257.

Super, D. E. (1942). The dynamics of vocational adjustment. New York: Harper \& Row.

Super, D. E. (1951). Vocational adjustment: Implementing a self-concept Occupations, 30, 88-92.

Super, D. E. (1953). A theory of vocational development. American Psychologist, 8, 185-190.

Super, D. E. (1955). The dimensions and measurement of vocational maturity. Teachers College Record, 57, 151-163.

Super, D. E. (1957). The psychology of careers. New York: Harper \& Row.

Super, D. E. (1963a). Vocational development in adolescence and early adulthood: Tasks and behavior. Em D. E. Super \& Cols. (Orgs.), Career development: Self concept theory (pp. 1-16). New York: College Entrance Examination Board.

Super, D. E. (1963b). Toward making self-concept theory operational. Em Super \& Cols. (Orgs.), Career development: Self concept theory (pp. 17-32). New York: College Entrance Examination Board.

Super, D. E. (1963c). Vocational development in adolescence and early childhood: Tasks and behavior. Em D. E. Super \& Cols. (Orgs.), Career development: Seff concept theory (pp. 79-95). New York: College Entrance Examination Board.
Super, D. E. (1969). Vocational development theory. The Counseling Psychologist, $1,2-30$.

Super, D. E. (1972). Vocational development theory in twenty years. Em J. M. Whiteley \& A. Resnikoff (Orgs.), Perspectives on vocational development. Washington, DC: American Personnel and Guidance.

Super, D. E. (1973). The career development inventory. British Journal of Guidance \& Counselling, 1, 37-50.

Super, D. E. (1977). Vocational maturity in mid-career. Vocational Guidance Quarterly, 25, 294-302.

Super, D. E. (1980). A life-time, life-space, approach to career development. Journal of Vocational Behavior, 13, 282-298.

Super, D. E. (1981). A developmental theory: Implementing a self-concept. Em D. H. Montross \& C. J. Shinkman (Orgs.), Career development in the 80s: Theory and practice. Springfield, IL: Charles C. Thomas.

Super, D. E. (1983). Assessment in career guidance: Toward truly developmental counseling. Personal and Guidence Journal, 61, 555-562.

Super, D. E. (1984). Career and life development. Em D. Brown, L. Brooks \& cols. (Orgs.), Career choice and development: Applying contemporary theories to practice. San Francisco: Jossey-Bass.

Super, D. E. (1985a). Exploration des frontières du développement vocationnel. Connat, 8, 271-297.

Super, D. E. (1985b). Coming of age in Middletown: Careers in the making. American Psychologist, 40, 405-414.

Super, D. E. (1990). The life-span, life-space approach to career development. Em D. Brown, L. Brooks \& cols. (Orgs.), Carreer choice and development (pp. 197-261) (2a ed.). San Francisco: Jossey-Bass.

Super D. E. \& Kidd, J. M. (1979). Vocational maturity in adulthood: Toward turning a model into a measure. Journal of Vocational Behavior, 14, 255-270.

Super, D. E., Osborne, W. L., Walsh, D. J., Brown, S. D. \& Niles, S. J. (1992). Developmental career assessment and counseling: The C-DAC. Journal of Counseling and Development, 71, 74-80.

Super, D. E. \& Overstreet, P. L. (1960). The vocational maturity of 9 th grade boys. New York: Teachers College Press.

Super, D. E., Savickas, M. L. \& Super, C. M. (1996). The life-span, life-space approach to careers. Em D. Brown, L. Brooks \& cols. (Orgs.), Career cboice and development (pp. 121-178) (3 $3^{\text {rd }}$ ed.). San Francisco: Jossey-Bass.

Super, D. E., Sverko, B. \& Super, C. M. (1995). Life roles, values and careers: International findings of the work, importance study. San Francisco: Jossey-Bass.

Super, D. E. \& Thompson, A. S. (1979). A six-scale, two factor measure of adolescent career vocational maturity. Vocational Guidance Quarterly, 28, 6-15.

Super, D. E., Thompson, A. S. \& Lindeman, R. H. (1988). Adult Concerns Inventory: Manual for research and exploratory use in counseling. Palo Alto, CA: Consulting Psychologists Press.

Super, D. E. \& Zelkowitz, R. (1974). Vocational maturation in the third decade of life: Definitions and measuring the confrontation of the tasks of professional development in the middle of the individual's career. Revista de Psicologia Generaly Aplicada, 29, 691-710.

Tiedeman, D. V. \& Miller-Tiedeman, A. (1984). Career decision making: An individualistic perspective. Em D. Brown, L. Brooks \& cols. (Orgs.), Career choice and development (pp. 281-310). San Francisco: Jossey-Bass.

Tiedeman, D. V. \& O'Hara, R. P. (1963). Career Development: Choice and adjustment. New York: College Entrance Examination Board.

Tilden, A. J. (1976). Is there a monotonic criterion for measures of vocational maturity in college students? Journal of Vocational Behavior, 12, 43-52.

Thomason, S. L. \& Winer, J. L. (1994). Career maturity and familial independence among college freshmen. Journal of Career development, 21, 23-35.

Ullery, J. W. \& O'Brien, R. K. (1970). Testing of the guidance program, project ABLE. Pittsburgh, PA: American Institutes for Research.

Vondracek, F. E., Lerner, R. M. \& Schulenberg, J. E. (1986). Career development: A life-span developmental approach. Hillsdale, NJ: Erlbaum. 
Watkins, C. E. \& Subich, L. M. (1995). Annual review, 1992-1994: Caree development, reciprocal work/non-work interaction, and woman's workforce participation. Journal of Vocational Behavior, 47, 109-163.

Watson, M. B., Stead, G. B. \& De Jager, A. C. (1995). The career development of black and white South African university students. International Journal for the Advanced of Counseling, 18, 39-47.

Ward, R. (1982). An assessment of the psychometric adequacy of the British adaptation of the Career Development Inventory. British Journal of Guidance $\&$ Counseling, 10, 185-194.

Westbrook, B. W. \& Mastie, M. M. (1973). Three measures of Vocational maturity: a beginning to know about. Measurement es Evaluation in Guidance, 6, 8-16.

Westbrook, B. W. \& Sanford, E. E. (1991). The validity of career maturity attitude measures among black and white high school students. The Career Development Quarterly, 39, 199-208.

Whiston, S. C. (1990). Evaluation in the Career Concerns Inventory. Journal of Counseling \& Development, 69, 78-80.
Young, R. A. (1984). Vocational choice and values in adolescent women. Sex Roles, 10, 485-492.

Young, R. A. \& Chen, C. P. (1999). Annual review: Practice and research in career counseling and development - 1998. The Career Development Quarterly, 48, 98141.

Recebido: 02/08/2002 $1^{a}$ Revisão: $10 / 12 / 2002$ Última Revisão: 11/03/200 Aceite Final: $17 / 03 / 2003$

Sobre o autor

Marcos Alencar Abaide Balbinotti é Ph.D. em Psicologia (Psicologia Geral) pela Université de

Montreal. É Professor e Pesquisador da Universidade do Vale do Rio dos Sinos. 